\title{
Stenting of superior vena cava and inferior vena cava for symptomatic narrowing after repeated atrial surgery for D-transposition of the great vessels
}

\author{
Pascal Chatelain, Bernhard Meier, Beat Friedli
}

\begin{abstract}
Double venous stenting of the superior vena cava and inferior vena cava was successfully performed after failed balloon angioplasty in a young woman who had venoatrial narrowing and kinking after repeated Mustard procedure for D-transposition of great vessels. Self-expanding metallic stents were used. Angiography showed that the first stent was fully patent after six months when a second stent was implanted. This operation was followed by sustained clinical improvement.
\end{abstract}

The present report shows the feasibility and success of stenting of the great veins when postoperative narrowing or kinking is unresponsive to balloon angioplasty alone.

The benefits of balloon angioplasty of elastic or fibrous postoperative venous lesions are often short-lived. Stenting of these vessels, initially tested by Maass and coworkers in the late $1970 \mathrm{~s},{ }^{1}$ is an alternative approach-as it is in various vascular beds. This technique, with or without balloon angioplasty, has been used in several clinical situations ${ }^{2-4}$ and small series. $^{56}$

Despite low velocity blood flow, stented veins seem to be able to remain patent. Long term patency of venous stents seems to be influenced by previous thrombosis or occlusion of the vesssel, ${ }^{2}$ but no series including long term follow up has been published until now.

Intimal proliferation with narrowing of the lumen within the device has been described as another consequence of stenting. This phenomenon seems to be more pronounced in veins than in arteries and is related to the stent design. Oversizing of the stent with stretching of the vessel could trigger this phenomenon. ${ }^{7}$

Obstruction of the superior vena cava and inferior vena cava is a classic complication after Mustard repair for transposition of the great vessels. ${ }^{89}$ Balloon dilatation may successfully relieve such stenoses. ${ }^{10}$

We report a case in which double venous stenting was used for recurrent postoperative narrowing of the superior vena cava and inferior vena cava at the venoatrial junction after an unsuccessful attempt at balloon dilatation.

\section{Case report}

A 17 year old girl had undergone balloon atrial septostomy (Rashkind manoeuvre) with good clinical improvement for D-transposition of the great vessels diagnosed at the age of 6 days.

At 4 months she showed clinical deterioration with hypoxia and convulsions and right hemiplegia secondary to a stroke developed. A palliative surgical atrial septostomy (BlalockHanlon operation) was performed without appreciable clinical improvement. Therefore, complete surgical correction with atrial reconstruction (Mustard operation) was carried out. A Dacron baffle was used.

The following 10 years were free of important cardiovascular events. An epicardial permanent pacemaker was implanted for sick sinus syndrome when she was 11 .

When she was 14 ankle oedema was seen and new cardiac investigations were performed. At catheterisation significant pressure gradients were found at the left pulmonary venoatrial junction $(13 \mathrm{~mm} \mathrm{Hg}$ ) and at the inferior cavoatrial junction $(7 \mathrm{~mm} \mathrm{Hg}$ ). Four months later clinical deterioration was seen with dyspnoea and recurrent haemoptysis. A new Mustard operation was performed rather than balloon angioplasty, which generally gives poor results in pulmonary venous obstructions. ${ }^{11}$ Bovine pericardium was used for the new baffle. Several weeks after the second surgical intervention generalised oedema and ascites developed, which were attributed to impaired systemic venous return. At catheterisation important narrowing of the superior vena cava and inferior vena cava was seen at the venoatrial junction. Balloon angioplasty was performed with moderate immediate success (residual gradients more than $3 \mathrm{~mm} \mathrm{Hg}$ ) because of the great elasticity of the venous walls.

At the age of 17 the patient was readmitted with recurrent severe generalised oedema and ascites. Catheter studies showed recurrent severe narrowing of the superior vena cava (gradient $12 \mathrm{~mm} \mathrm{Hg}$ ) and moderate narrowing of the inferior vena cava (gradient $8 \mathrm{~mm} \mathrm{Hg}$ ). Injection of contrast medium into the superior vena cava showed that there was no flow through the venoatrial junction and there were collateral vessels to the inferior vena cava and right atrium through the azygos vein, which appeared to be dilated (fig 1A). Injection of contrast into the inferior vena cava 
Figure 1 Angiogram of the superior vena cava showing $(A)$ lack of flow at the cavoatrial junction and collateralisation through the azygos vein (lateral view) and $(B)$ good passage of contrast medium through the stent and disappearance of collateralisation.

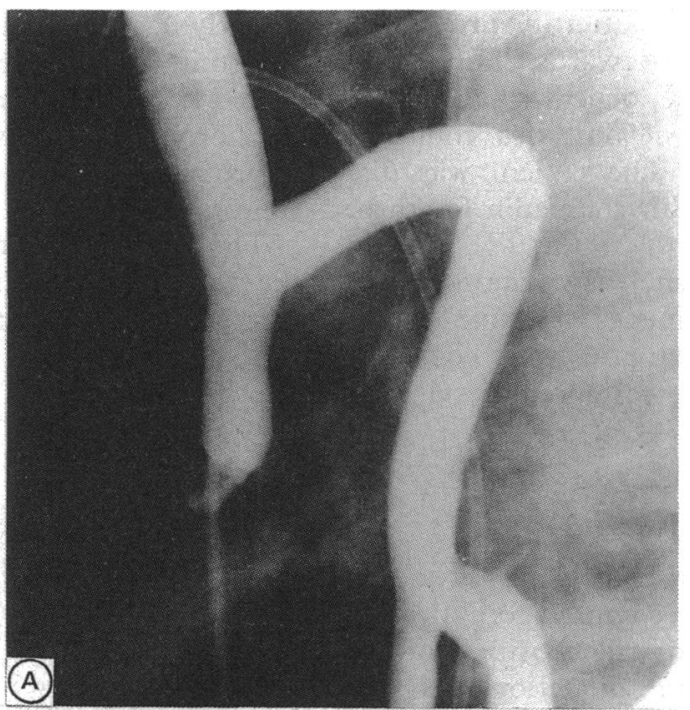

showed dilatation of that vessel' and suprahepatic veins and significant narrowing of the venoatrial junction (fig $2 \mathrm{~A}$ ).

For the interventional procedure, a new femoral venous access was established with a $14 \mathrm{~F}$ short introducer (Schneider). A steerable 0.035 inch J exchange $6 \mathrm{~cm}$ flexible tip "backup" guide wire (Schneider) was introduced into the superior vena cava. This allowed easy positioning of a $3 \times 9 \mathrm{~mm}$ trefoil balloon catheter (inflated diameter about $16 \mathrm{~mm}$ ). Four successive balloon inflations at $4,8,8$, and 9 atmospheres were performed. A persistent pressure gradient of $8 \mathrm{~mm} \mathrm{Hg}$ between the superior vena cava and the right atrium persisted but there was angiographic improvement. We decided to stent the narrowing. This was achieved with a 9F Wallstent (Schneider) (unconstrained diameter $16 \mathrm{~mm}$; length of $40 \mathrm{~mm}$ ). This system consists of a stainless steel multifilament, self-expanding, flexible, macroporous stent that is constrained by a doubled-over membrane and mounted on the delivery catheter. Heparin (2000 IU) was injected intraveneously before the stent was correctly positioned and the constraint released under fluoroscopic control. The stent was further dilated with the previously used trefoil balloon inflated to 9 atmospheres. Angiography showed a satisfactory result (fig 1 B) and the final pressure gradient between the superior vena cava and the right atrium was 1$2 \mathrm{~mm} \mathrm{Hg}$.

A first attempt at dilatation of the inferior vena cava with the $3 \times 9$ trefoil balloon produced no improvement. Further dilatation was then performed with a $10 \mathrm{~F} 3 \times 12$ balloon (inflated diameter about $20 \mathrm{~mm}$ ) introduced through a $16 \mathrm{~F}$ introducer. Two inflations at 5 and 6 atmospheres (balloon ruptured) produced some angiographic improvement but the pressure gradient was still $5 \mathrm{~mm} \mathrm{Hg}$. There was clinical improvement in the next few days and the patient was discharged and treated with low dose diuretics and oral anticoagulant for three months.

Six months later lower body oedema and ascites recurred. There were no clinical signs of obstruction of the superior vena cava. Angiographic assessment of the stent placed six months earlier in the superior vena cava showed that it was still in place at the venoatrial junction and that it was patent. The pressure gradient at this level was only $1 \mathrm{~mm}$ Hg. Angiography showed that the inferior vena cava, dilated six months earlier, was significantly narrowed and that there was a 7 $\mathrm{mm} \mathrm{Hg}$ pressure gradient. The vessel seemed to be kinked rather than circumferentially narrowed (fig 2, A). We decided to stent the inferior vena cava. A Wallstent identical to the one in the superior vena cava was positioned in the inferior venoatrial junction without prior balloon angioplasty. After release, dilatation of the stent with a trefoil balloon $3 \times 10 \mathrm{~mm}$ (inflated diameter about $17 \mathrm{~mm}$ ) at 5 atmospheres allowed further widening and shortening of the stent. Angiography showed a successful result (fig $2 \mathrm{~B}$ ). There was no 
residual pressure gradient after the procedure. The patient did well afterwards. Three days later ascites and ankle oedema had disappeared and the dose of diuretics could be considerably reduced. The patient was discharged and treated with coumadin for three months.

Five months later she was symptom free and had no signs of impeded systemic venous return. Her physical activities were considerably improved and she was about to start her first job.

\section{Discussion}

Narrowing of the superior vena cava and inferior vena cava is a recognised complication after Mustard repair of transposition of the great vessels. Balloon dilatation can be successful, but because it failed in the present case we decided to implant a stent.

This case of recurrent important postoperative narrowing of the vena cava gave useful information on the feasibility and outcome of balloon angioplasty and stenting of large veins. Immediate elastic recoil of venous tissue is invariably seen after balloon angioplasty but in this case the non-distensibility of the bovine pericardium used for Mustard repair may also have contributed. Though the narrowing of the superior vena cava was clearly circumferential that of the inferior vena cava seemed to be mainly caused by kinking. This may explain why the intrinsic expansion force of the stent opened up the narrowing. None the less, balloon dilatation of the stent further increased the diameter.

The excellent patency of the stent implanted six months earlier in our patient suggests that the risk of subsequent thrombosis or narrowing is low. Angiographic follow up of the second stent is not available but the considerable and sustained clinical improvement more than five months after implantation probably indicates patency of the device.

A review of reported small series ${ }^{245}$ and cases $^{12-14}$ of venous stenting shows that the underlying anatomical conditions partly determine the long term patency of the device. Narrowing of the vessel without an underlying fibrotic, thrombotic, or infiltrative process is probably the best condition for long term stenting.

The implantation of a stent may prove to be the best treatment for symptomatic venous narrowing after Mustard operation.

1 Maass D, Kropf L, Egloff L, Demierre D, Turina M, Senning A. Transluminal implantation of intravascular "double helix" spiral prostheses: technical and biologica considerations. Proc Eur Soc Artif Organs 1982;2:252-6.

2 Charnsangavej C, Carrasco CH, Wallace S, et al. Stenosis o the vena cava: preliminary assessment of treatment with expandable metallic stents. Radiology $1986 ; 61: 295-8$.

3 Wright KC, Wallace S, Charnsangavej C, Carrasco CH, Gianturco C. Percutaneous endovascular stents: an experimental evaluation. Radiology 1985;156:69-72.

4 Gunther RW, Vorwerk D, Bohndorf $\mathrm{K}$, et al. Venous stenoses in dialysis shunts: treatment with self-expanding stenoses in dialysis shunts: treatment with
metallic stents. Radiology 1989;170:403-5.

5 Zollikofer CL, Largiader I, Bruhlmann WF, Uhlschmid GK, Marty A. Endovascular stenting of veins and grafts: preliminary clinical expeerience. Radiology 1988;167:

6 Mullins CE, O'Laughlin MP, Wesley Vick G III, et al. Implantation of balloon-expandable intravascular grafts by catheterization in pulmonary arteries and systemic veins. Circulation 1988;77:188-99.

7 Duprat G Jr, Wright KC, Charnsangavej C, Wallace S Gianturco C. Self-expanding metallic stents for small vessels: an experimental evaluation. Radiology 1987; 162:469-72.

8 Kron IL, Rheuban KS, Joob AW, et al. Baffle obstruction following the Mustard operation: cause and treatment. Ann Thorac Surg 1985;39:112-5.

9 Darvell FJ, Rossi IR, Rossi MB, et al. Intermediate to late term results of Mustard's procedure for complete transposition of the great arteries with an intact ventricular septum. Br Heart J 1988;59:468-73.

10 Benson LN, Yeatman L, Laks H. Balloon dilatation for superior vena caval obstruction after the Senning procedure. Cathet Cardiovasc Diagn 1985;11:63-8.

11 Cooper SG, Sullivan ID, Bull C, Taylor JFN. Balloon dilation of pulmonary venous pathway obstruction after Mustard repair for transposition of the great arteries. J Am Coll Cardiol 1989;14:194-8.

12 Odell JA, Shama D, Crause L. Retained knife blade and acute superior vena cava obstruction. J Trauma 1988 28:416-7.

13 Barnes RW, Fleisher HL III, Redman JF, Smith JW, Harshfield DL, Ferris EJ. Mesoaortic compression of the left renal vein: repair by a new stenting procedure. $J$ Vasc Surg 1988;8:415-21

14 Gunther RW, Vorwerk D, Klose KC, et al. Self-expanding stents for the treatment of a long venous stenosis in a dialysis shunt: case report. Cardiovasc Intervent Radiol 1989;12:29-31. 